\title{
La conexión neumocóccica
}

\author{
Sergio Mella, Gisela Riedel, Loreto Twele, Mariana Domínguez, Helia Bello y Gerardo González
}

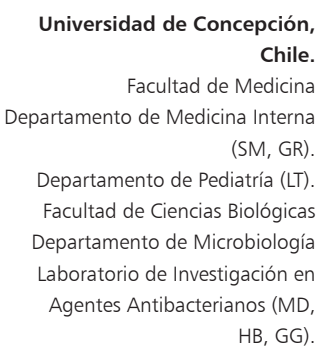

Declaración de conflictos de interés: Sergio Mella ha recibido honorarios por conferencias y financiamiento para asistir a congresos de Astra-

Zeneca y Merck Sharp Dohme; honorarios por asesorías de Pfizer y Novartis.

Fuente de financiamiento: Trabajo financiado por Proyecto DIUC 205.085.029-1.0

Recibido: 24 de septiembre de

Aceptado: 27 de noviembre de

Correspondencia a: Sergio Mella Montecinos pignatio@vtr.net
U no de los puntos críticos actuales en el área de las enfermedades infecciosas es la falta de alternativas terapéuticas útiles y seguras para el tratamiento de infecciones producidas por bacilos gramnegativos resistentes a carbapenémicos ${ }^{1-3}$. Sin duda, esta clase de antimicrobianos $\beta$-lactámicos ha marcado un hito importante en la historia de la quimioterapia antibacteriana moderna ya que constituyen los agentes de elección para el tratamiento de infecciones producidas por cepas de estas bacterias resistentes a cefalosporinas de tercera generación ${ }^{4,5}$. Es precisamente en este contexto -infecciones por enterobacterias portadoras de $\beta$ lactamasas de espectro extendido (BLEE) o cefalosporinasas tipo Amp C-donde no se ha puesto suficiente énfasis en el impacto que ha tenido en su desarrollo el sobreuso de cefalosporinas de tercera generación, particularmente ceftriaxona, la cual es utilizada sistemáticamente en el tratamiento de múltiples tipos de infecciones (como urinarias, respiratorias e incluso de tejidos blandos).

Es interesante recordar que uno de los argumentos más frecuentemente encontrados en la literatura médica para promover el uso de cefalosporinas de tercera generación fue la descripción de susceptibilidad disminuida de Streptococcus pneumoniae a penicilina ${ }^{6-9}$.

Cuando uno de los autores estudiaba Medicina pudo apreciar cómo pacientes con un cuadro de neumonía lobar con fiebre, escalofríos, expectoración herrumbrosa, puntada de costado con tope inspiratorio y hallazgos semiológicos clásicos como el soplo tubario, respondían favorablemente al uso de penicilina $G$ sódica por vía intramuscular con dosis -que a la luz actual- resultan sorprendentemente bajas. Ya a fines de los 70 comenzaron a aparecer las primeras descripciones de cepas de $S$. pneumoniae con alteración en la composición de sus PBPs y/o en la actividad autolítica; este fenómeno fue creciendo y globalizándose, alcanzando algunos clones distribución primero continental y luego mundial ${ }^{6-9}$.

Ese fue el factor principal para la utilización -cada vez más sistemática y avalada posteriormente por recomendaciones internacionales y particularmente estadounidenses- ${ }^{10-12}$ de cefalosporinas de tercera generación como agentes de elección para el tratamiento de infecciones neumocóccicas invasoras ${ }^{10-12}$. Debemos contextualizar que, al momento de su desarrollo por la industria farmacéutica, el foco de actividad de estas moléculas estaba centrado sobre bacilos gramnegativos resistentes a otros $\beta$-lactámicos ${ }^{13,14}$. El favorable perfil farmacocinético de ceftriaxona facilitó aún más su utilización: su prolongada vida media permitió su utilización una vez al día, exceptuando infecciones del $\mathrm{SNC}^{13,14}$. Incluso fueron publicados una serie de análisis que enfatizaron la perspectiva farmacoeconómica de tal perfil farmacocinético, al permitir ahorrar insumos y tiempo de enfermería ${ }^{15,16}$. A poco andar, este tipo de agentes antibacterianos, orientados inicialmente para el tratamiento de infecciones por bacilos gramnegativos, se convirtieron en agentes de elección en el tratamiento de un patógeno grampositivo clásico, agente etiológico de múltiples infecciones particularmente comunitarias y muchas de ellas asociadas a una elevada morbimortalidad ${ }^{17,18}$. Sin embargo, este uso sistemático, en forma silente y sostenida 
se asoció a un hecho que estaba indefectiblemente ligado a uno de los principios básicos de la terapia antibacteriana: la acción de los antimicrobianos es sobre blancos típicos de la célula bacteriana, un procarionte, ausentes por tanto de las células eucarióticas de nuestro organismo ${ }^{19,20}$. El concepto de "bala mágica" esto es "encontrar un medio que mate al parásito en el cuerpo, sin tener un efecto perjudicial sobre éste" que explicaba la especificidad y selectividad de los antibacterianos y por tanto que hace a la quimioterapia antibacteriana tan eficaz, potente y poco tóxica, implicaba que el antimicrobiano no sólo destruía las bacterias que producían infección en el pulmón, sino que también, producía un efecto no menor, sobre la totalidad del microbioma del paciente en tratamiento. Nuestra microbiota, actualmente denominada microbioma, presente en mucosas, piel y particularmente a nivel intestinal, representa un sistema tan complejo y vasto, que sólo en número resulta superior en uno o dos órdenes de magnitud en comparación a las células eucarióticas de nuestro cuerpo ${ }^{21-25}$.

Este microbioma fue lenta y sostenidamente sometido a la presión selectiva ejercida por las cefalosporinas de tercera generación, utilizadas cada vez en forma más frecuente, especialmente sobre enterobacterias que en general albergan ya $\beta$-lactamasas parentales como TEM1, TEM-2 y SHV-1. De esta forma se vieron favorecidos diversos fenómenos genéticos que explican el desarrollo de resistencia, especialmente mutaciones, produciendo finalmente bacilos gramnegativos resistentes a cefalosporinas de tercera generación ${ }^{26-28}$ (Figura 1).

En la presente discusión se debe enfatizar que la diferencia entre una $\beta$-lactamasa de reducido espectro, ya sea de estirpe TEM o SHV, de una de espectro extendido radica en cambios en sólo uno o pocos aminoácidos que alteran la configuración del sitio activo de la enzima, pudiendo de este modo hidrolizar ahora cefalosporinas de tercera generación y monobactámicos ${ }^{28-30}$. Dancer, en un artículo poco valorado, señaló que el problema principal de las cefalosporinas es la presión que ejercen para el desarrollo de, a lo menos, bacilos gramnegativos resistentes a estos agentes: fuese por la producción de $\beta$-lactamasas de espectro extendido y/o por otro marcador evidente del sobreuso de estos antimicrobianos: Enterobacter spp. y producción de cefalosporinasas, además de propiciar otros patógenos de especial importancia actual como Enterococcus spp. y Clostridium difficile ${ }^{31,32}$. Si se evalúa la lista de los patógenos incluidos bajo el acrónimo ESKAPE, la mitad de ellos han emergido, al menos en parte, favorecidos por el sobreuso de cefalosporinas, particularmente las de tercera generación ${ }^{33}$.

Para enfatizar sobre los puntos anteriormente discutidos recordemos que, al utilizar ceftriaxona, al menos $40 \%$ de la dosis administrada es eliminada en la bilis, favoreciendo el fenómeno de presión selectiva sobre el microbioma intestinal ${ }^{34,35}$.
Entonces, en el tratamiento de neumonía adquirida en la comunidad, las cefalosporinas de tercera generación se convirtieron en el estándar de tratamiento, sin importar los hallazgos microbiológicos que muchas veces demostraban aislados susceptibles a múltiples antibacterianos que ejercían menor presión selectiva o daño ecológico; la antonomasia de esta conducta fue el planteamiento por destacados especialistas estadounidenses que no era necesario solicitar hemocultivos en el estudio de un enfermo con neumonía, dado que esta información no era utilizada ${ }^{36,37}$. Para complicar aún más el problema de sobreuso de estos agentes, ahora aprovechando su favorable acción sobre bacilos gramnegativos, estos antibacterianos se convirtieron, de hecho, en agentes de primera línea en el tratamiento de infecciones adquiridas en la comunidad, tales como la peritonitis secundaria; así, independientemente de la juventud del paciente y la ausencia de co-morbilidades, y hallazgos favorables durante la apendicectomía (apéndice sin perforación, con escaso líquido peri apendicular) éste recibe ceftriaxona más un agente antianaeróbico; similar situación ocurre en infecciones del tracto urinario alto: aquella paciente joven con un episodio de pielonefritis aguda sin elementos de sepsis grave recibe ceftriaxona de igual manera, a pesar de función renal conservada e incluso aislamiento de un patógeno susceptible a un aminoglucósido en orina, -situación frecuente en nuestro medio- ${ }^{38}$. Incluso en infecciones de piel y tejidos blandos, aparte del uso de cefalosporinas de primera generación, se utiliza ceftriaxo$\mathrm{na}^{39,40}$; un ejemplo frecuente es de pacientes con un cuadro clínico absolutamente compatible con una erisipela y el paciente, de manera independiente si está siendo tratado en el ámbito público o privado recibirá en muchos centros, ceftriaxona en vez de penicilina, las explicaciones son

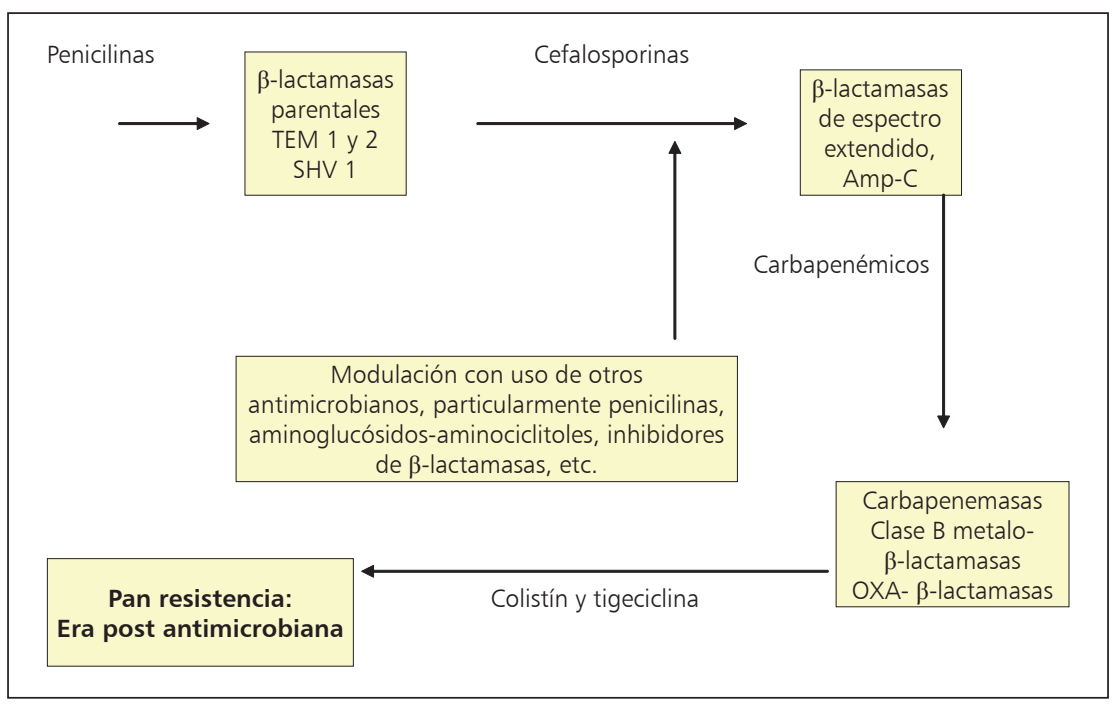

Figura 1. Modelo del sobreuso de cefalosporinas y generación de resistencia. 
múltiples, pero ante la pregunta ¿por qué en una infección estreptocóccica probablemente producida por especies de Streptococcus spp. altamente susceptibles a penicilina, se utiliza ceftriaxona? La respuesta fundamental es la comodidad de la administración de una dosis diaria; no hay ninguna consideración microbiológica sobre el escenario de resistencia actual y el efecto de esta molécula en particular sobre nuestro microbioma ${ }^{41-45}$.

Además, el sostenido incremento de resistencia en Neisseria gonorrhoeae a quinolonas y la falta de otras alternativas terapéuticas ha inducido a considerar el uso de cefalosporinas de tercera generación como agentes de elección para el tratamiento de infecciones producidas por este patógeno clásico de transmisión sexual ${ }^{46,47}$.

Peor aún, el uso de ceftriaxona no se ha limitado al ámbito del tratamiento de infecciones adquiridas en la comunidad, sino que incluso se ha expandido como agente antibacteriano utilizado en profilaxis quirúrgica -principalmente por la farmacocinética de la molécula y confusión entre profilaxis y tratamiento- ${ }^{48,49}$.

Si consideramos que muchas de las infecciones comentadas son causa frecuente de consulta y hospitalización, la pregunta siguiente y que corresponde a un lugar común - pero que a nuestro criterio es sumamente válido- ¿Qué sucedería en cualquier hospital de alta complejidad si en Farmacia no hay disponibilidad de cefalosporinas de tercera generación? Interesantemente en muchas de las situaciones planteadas existen otras alternativas terapéuticas que podrían jugar un rol no menor en la modulación de la resistencia, particularmente hoy donde el problema fundamental es la resistencia en bacilos gramnegativos.

Así, ya hemos planteado que el uso de antimicrobianos tradicionales todavía tiene un papel importante en nuestros días; por ejemplo, una penicilina de reducido o de amplio espectro incidirá en una menor presión selectiva comparada con una cefalosporina de tercera generación en la selección de cepas resistentes a nivel intestinal. En general, las primeras se asociarán a la selección de aislados productores de $\beta$-lactamasas parentales, tales como TEM-1, TEM-2 y SHV-1.

Otro punto crucial es la utilidad de agentes como los aminoglucósidos-aminociclitoles, considerados por algunos especialistas como de utilidad principalmente en países subdesarrollados y como agentes poco seguros y de eficacia discutible ${ }^{50,51}$. Estas moléculas ejercen una fuerte acción bactericida sobre bacilos gramnegativos aeróbicos, producen una menor presión selectiva comparadas con las cefalosporinas de tercera generación, particularmente por su ausencia de actividad antimicrobiana sobre la microbiota anaerobia; y sí constituyen agentes confiables y seguros en la práctica clínica habitual en nuestro país, especialmente cuando son utilizadas en función de las características del paciente. En este marco debe insistirse que no se recomienda el uso de estos agentes en pacientes añosos, deshidratados y en los cuales se está utilizando al menos otro agente nefrotóxico. Un ejemplo típico en el cual no deben usarse estos agentes sería la de una paciente de 68 años que fue intervenida por una peritonitis secundaria, sin monitorización de su intravascular, ni diuresis y que además está recibiendo para el manejo del dolor AINEs. El epítome de este contexto sería la realización de un tomografía con contraste para descartar una colección.

La antítesis del ejemplo anterior es el poco uso de estos agentes en infecciones del tracto urinario alto en pacientes jóvenes, donde deben considerarse agentes de elección y donde de hecho existen estudios prospectivos que demuestran la utilidad de estos agentes en ese marco clínico $^{51}$.

Antes de finalizar resulta atingente revisar cuál es la situación de susceptibilidad disminuida a penicilina de S. pneumoniae en nuestro país. Datos analizados por el Grupo Colaborativo de Resistencia Antimicrobiana, que reúne a 14 hospitales, revelan la baja tasa de resistencia a penicilina: de 176 cepas aisladas de líquidos estériles (no LCR) durante el año 2009 en adultos, sólo una resultó con susceptibilidad intermedia a penicilina y ninguna resistente. En población pediátrica la situación es similar, sólo 3 cepas fueron catalogadas como con susceptibilidad intermedia y ninguna resistente, de un total de 72 aislados $^{52}$. La consolidación del análisis de la vigilancia nacional de laboratorio de infecciones neumocóccicas realizada por el Instituto de Salud Pública ${ }^{53}$ muestra que entre los años 2007 y 2011, 98\% de las cepas no meníngeas y $71 \%$ de las meníngeas fueron susceptibles a penicilina (total 755 aislados). Al clasificar la susceptibilidad de las cepas según grupos etarios, los resultados son similares salvo en el caso de cepas obtenidas de niños bajo 5 años de edad, con diagnóstico de meningitis (12 de 20 cepas resultaron resistentes).

A modo de conclusión planteamos que, dado que el fenómeno del desarrollo de resistencia bacteriana a los antimicrobianos es un fenómeno biológicamente inevitable pero si modulable ${ }^{54}$, debe enfatizarse que antibacterianos tradicionales y del menor espectro posible tienen aún un papel crucial en el tratamiento de una serie de infecciones $^{18,42,51}$ (Tabla 1) y que el uso de antimicrobianos no debe ser basado fundamentalmente en la facilidad de su administración, sino que debe contrastarse con la probabilidad de ejercer una gran presión selectiva sobre el microbioma humano de acuerdo a su espectro de acción.

\section{Resumen}

La resistencia de los bacilos gramnegativos constituye una de las áreas de mayor importancia en la medicina moderna; sin embargo, no se ha destacado el papel preponderante de las cefalosporinas de tercera generación y 
Comentario: neumonía que requiere hospitalización en sala de Medicina, * propuesta hecha por ConsenSur $11^{55} ;{ }^{* *}$ recomendación australiana ${ }^{56}$; debe considerarse puntos de corte actuales del CLSI para infecciones extrameníngeas ${ }^{57}$ y por tanto mayor énfasis en la posibilidad de utilizar penicilina cuando se obtenga confirmación de etiología neumocóccica 57,18

\section{Pielonefritis aguda}

Aminoglucósidos-aminociclitoles

Se recomienda revisar artículo de Leibovici y col$^{51}$

\section{Peritonitis secundaria}

\section{Aminoglucósido-aminociclitol + metronidazol}

Tema controversial, si bien consensos estadounidenses y canadienses recomiendan esquemas que no incluyen aminoglucósidos-aminociclitoles por menor eficacia y toxicidad 58,59 , al revisar meta-análisis que apoyan esta postura se observa mortalidades equivalentes al comparar esquemas con aminoglucósidos más agente antianaeróbico versus comparadores ${ }^{60,61}$, lo mismo una revisión sistemática que además sugiere en sus conclusiones que deben considerarse guías locales y costos junto con nuevos estudios que logren estratificar a los diferentes grupos de pacientes ${ }^{62}$. De lo anterior se deriva que, en pacientes adultos jóvenes, sin injuria renal, ni shock séptico, la asociación de gentamicina en monodosis diaria más metronidazol es una alternativa válida, particularmente considerando que la resolución del cuadro es quirúrgica y que la terapia antimicrobiana es sólo coadyuvante

4. Infección cervico-facial

Penicilina a dosis elevada más metronidazol

Origen odontogénico u otorrinolaringológico, se excluyen cuadros de etiología principalmente estafilocóccica como celulitis orbitaria o trombosis supurativa de seno cavernoso40
5. Absceso cerebral
Penicilina a dosis elevada más metronidazol

Infecciones polimicrobianas, en las cuales el drenaje quirúrgico es el elemento principal de la terapia

\section{Celulitis en pacientes inmunocompetentes}

Cloxacilina

A pesar de lo señalado por líderes de opinión estadounidenses que favorecen el uso de ceftriaxona ${ }^{61}$, nuestra postura enfatiza uso de isoxazolil penicilinas. Un reciente estudio demuestra equivalencia entre monoterapia con flucloxacilina versus flucloxacilina más benzilpenicilina ${ }^{64}$
7. Erisipela
Penicilina

Infección estreptocóccica extrameníngea

\section{Profilaxis quirúrgica}

Cefazolina

Insistir en uso tradicional de cefazolina para la mayoría de las cirugías ${ }^{65}$

particularmente de ceftriaxona en la selección de bacilos gramnegativos resistentes a estos agentes. Paradójicamente, ceftriaxona -al igual que el resto de las moléculas de esta generación- cuya indicación inicial fueron las infecciones por bacilos gramnegativos comenzó a utilizarse como agente de elección en infecciones neumocóccicas. El amplio espectro de actividad de esta molécula junto a sus favorables propiedades farmacocinéticas hicieron que este antimicrobiano reemplazara a otros agentes en el tratamiento de un amplio número de infecciones comunitarias. No obstante, no se consideró la acción de esta cefalosporina sobre el microbioma, especialmente intestinal, que permitió la selección de enterobacterias que mediante eventos genéticos, especialmente mutaciones de $\beta$-lactamasas parentales (TEM-1, TEM-2, SHV-1), desarrollaron ahora resistencia a cefalosporinas de tercera generación. De esta forma la disminución de susceptibilidad a penicilina en aislados de Streptococcus pneumoniae que estimuló el uso creciente de ceftriaxona, fue uno de los principales impulsores para el desarrollo de resistencia a cefalosporinas de tercera generación en bacilos gramnegativos.

\section{Referencias bibliográficas}

1.- Livermore D M. Has the era of untreatable infections arrived? J Antimicrob Chemother 2009; 64 (Supp 1): 29-36.

2.- Miyakis S, Pefanis A, Tsakris A. The challenges of antimicrobial drug resistance in Greece. Clin Infect Dis 2011; 53: 177-84.

3.- Burgess D S, Rapp R P. Bugs versus drugs: Addressing the pharmacist's challenge. Am J Health-Syst Pharm 2008; 65 (Suppl 2): S4-S15.

4.- Tumbarello M, Sanguinetti M, Montuori E, Trecarichi E M, Posteraro B, Fiori B, et al.
Predictors of mortality in patients with bloodstream infections caused by extendedspectrum-ß-lactamases-producing Enterobacteriaceae: Importance of inadequate initial antimicrobial treatment. Antimicrob Agents Chemother 2007; 51: 1987-94.

5.- $\quad$ Yang K, Guglielmo B J. Diagnosis and treatment of extended-spectrum and Amp-C betalactamase producing organism. Ann Pharmacother 2007; 41: 1427-35.

6.- Jacoby G A. Prevalence and resistance mechanisms of common bacterial respiratory pathogens. Clin Infect Dis 1994; 18: 951-7.
7.- Klugman K P. Pneumococcal resistance to antibiotics. Clin Microbiol Rev 1990; 3: 171-96.

8.- Appelbaum P C. Antimicrobial resistance in Streptococcus pneumoniae: an overview. Clin Infect Dis 1992; 15: 77-83.

9.- Bergmann C, Chi F, Rachid S, Hakenbeck R. Mechanisms for penicillin resistance in Streptococcus pneumoniae: penicillin-binding proteins, gene transfer and cell wall metabolism. Tuomanen EI, Mitchell TJ, Morrison DA, Spratt BG, eds. The pneumococcus. Washington, DC: ASM Press American Society for Microbiology; 2004, p. 339-49. 
10.- Mandell L A, Wunderink R G, Anzueto A, Bartlett J G, Campbell G D, Dean N C, et al. Infectious Diseases Society of America/ American Thoracic Society Consensus Guidelines on the management of communityacquired pneumonia in adults. Clin Infect Dis 2007; 44 (Suppl 2): S27-S72.

11.- Mandell L A, Marrie T, Grossman R F, Chow A W, Hyland R H, et al. Canadian guidelines for initial management of community acquired pneumonia: an evidence based update by the Canadian Infectious Diseases Society and Canadian Thoracic Society. Clin Infect Dis 2000; 31: 383-421.

12.- Niederman M S, Mandell L A, Anzueto A, Bass J B, Broughton W A, Campbell G D, et al. American Thoracic Society. Guidelines for the management of adults with community acquired pneumonia. Diagnosis, assessment of severity, antimicrobial therapy, and prevention. Am J Respir Crit Care Med 2001; 163: 1730-54.

13.- Donowitz G R, Mandell G L. Beta-lactam antibiotics. N Engl J Med 1988; 318: 490-500.

14.- Richards D M, Heel R C, Brogden R N, Speight T M, Avery G S. Ceftriaxone. A review of its antibacterial activity, pharmacological properties and therapeutic use. Drugs 1984; 27 : 469-527.

15.- Balant L, Dayer P, Auckenthaler R. Clinical pharmacokinetics of the third generation cephalosporins. Clin Pharmacokinetics 1985; 10: 101-43.

16.- Adu A, Armour CL. Drug utilization review (DUR) of the third generation cephalosporins. Drugs 1995; 50: 423-36.

17.- Musher D M. Streptococcus pneumoniae. Mandell GL, Bennett JE, Dolin R, editors. Mandell, Douglas and Bennett's Principles and Practice of Infectious Diseases, 7th ed. Philadelphia: Churchill Livingstone Elsevier; 2010, p. 2623-42.

18.- Mella S, Blamey R, Paiva O, Yáñez J, Riedel G, Aylwin M, et al. Impacto de la evaluación infectológica en el uso racional de antimicrobianos en pacientes con bacteriemia por S. pneumoniae. Rev Chilena Infectol 2007; 24: 264-9.

19.- Calvo A. Ehrlich y el concepto de "bala mágica”. Rev Esp Quimioterap 2006; 19: 90-92.

20.- García-Sánchez J, García E, Merino M. Cien años de la bala mágica del Dr. Ehrlich (19092009). Enferm Infecc Microbiol Clin 2010; 28: 521-33.

21.- Jernberg C, Lofmark S, Edlund C, Jansson J K. Long-term impacts of antibiotic exposure on the human intestinal microbiota. Microbiology 2010; 3216-23.

22.- Dethlefsen L, Relman D A. Incomplete recovery and individualized responses of the human distal gut microbiota to repeated antibiotic perturbation. Proc Natl Acad Sci U S A 2011; 108 Suppl 1: S4554-S61.
23.- Donskey C. The role of the intestinal tract as a reservoir and source for transmission of nosocomial pathogens. Clin Infect Dis 2004; 39 : 219-26.

24.- New Perspectives Quarterly 2010; 27 (4). Disponible en: http://www.digitalnpq.org/ global_services/nobel\%20laureates/04-21-03. html.

25.- Baquero F, Coque TM, de la Cruz TM. Ecology and evolution as targets : the need for novel eco-evo drugs and strategies to fight antibiotic resistance. Antimicrob Agents Chemother 2011; 55: 3649-60

26.- Livermore DM. $\beta$-lactamase-mediated resistance and opportunities for its control. J Antimicrob Chemother 1998; 41(Suppl. D): S25-S41.

27.- Jacoby G A, Medeiros A A. More extendedspectrum $\beta$ lactamases. Antimicrob Agents Chemother 1991; 35: 1697-704.

28.- Opal S M, Pop-Vicas A. Molecular mechanisms of antibiotic resistance in bacteria. Mandell, Douglas and Bennett's Principles and Practice of Infectious Diseases, 7th ed. Mandell GL, Bennett JE, Dolin R, eds. Philadelphia: Churchill Livingstone Elsevier; 2010 p. 279-95.

29.- Philippon A, Labia R, Jacoby G. Extendedspectrum $\beta$-lactamases. Antimicrob Agents Chemother 1989; 33: 1131-6.

30.- Sougakoff W, Gaussard S, Courvalin P. The TEM-3-lactamase, wich hydrolizes broad spectrum cephalosporins, is derived from the TEM-2 penicillinase by two amino acid substitutions. FEMS Microbiol Lett 1988; 56: 343-8.

31.- Dancer S J. The problem with cephalosporins. J Antimicrob Chemother 2001; 48: 463-78.

32.- Chow J W, Fine M J, Shlaes D M, Quinn J P, Hooper D C, Johnson M P, et al. Enterobacter bacteremia: clinical features and emergence of antibiotic resistance during therapy. Ann Intern Med 1991; 115: 585-90.

33.- Boucher H W, Talbot G H, Bradley J S, Edwards J E, Gilbert D, Rice L B, et al. Bad bugs, no drugs: No ESKAPE! An Update from the Infectious Diseases Society of America. Clin Infect Dis 2009; 48: 1-12.

34.- Bryskier A, Aszodi J. Cephems for parenteral use. In Bryskier A, editor. Washington, DC: ASM Press American Society for Microbiology 2005; p: 163-221.

35.- Andes D R, Craig W A. Cephalosporins. In: Mandell, Douglas and Bennett's Principles and Practice of Infectious Diseases, Mandell GL, Bennett JE, Dolin R, eds. 7th ed. Philadelphia: Churchill Livingstone Elsevier; 2010 p. 323-39.

36.- Waterer G W, Jennings S G, Wunderink R G. The impact of blood cultures on antibiotic therapy in pneumococcal pneumonia. Chest 1999; 116: 1278-81.

37.- Campbell S G, Marrie T J, Anstey R,
Dickinson G, Ackroyd-Stolarz S. The contribution of blood cultures to the clinical management of adult patients admitted to the hospital with community-acquired pneumonia. A prospective observational study. Chest 2003; 123: 1142-50.

38.- Valdivieso F, Trucco O, Prado V, Díaz M C, Ojeda A. Resistencia a los antimicrobianos en agentes causantes de infección del tracto urinario en 11 hospitales chilenos. Proyecto PRONARES. Rev Med Chile 1999; 127: 1033-40.

39.- Bradsher R W Jr, Snow R M. Ceftriaxone treatment of skin and soft tissue infections in a once daily regimen. Am J Med 1984; 77: 63-7.

40.- Swartz MN. Cellulitis. N Engl J Med 2004; 350 : 904-12.

41.- Goldstein E J C. Beyond the target pathogen: ecological effects of the hospital formulary. Curr Opin Infect Dis 2011; 24 (Suppl 1): S21-S31.

42.- Mella S, Muñoz M. Utilidad de penicilina G en infecciones estreptocócicas. Rev Med Chile 2009; 137: 585-6.

43.- Fujitani S, Rowlinson M C, George W L. Penicillin G-resistant viridans group streptococcal endocarditis and interpretation of the American Heart Association's Guidelines for the Treatment of Infective Endocarditis. Clin Infect Dis 2008; 46: 1064-6.

44.- Climo M W, Israel D S, Wong E S, Williams D, Coudron P, Markowitz S M. Hospital-wide restriction of clindamycin: effect on the incidence of Clostridium difficile-associated diarrhea and cost. Ann Intern Med 1998; 128: 989-95.

45.- Paterson D L. "Collateral damage" from cephalosporin or quinolone antibiotic therapy. Clin Infect Dis 2004; 38 (Suppl 4): S341-5.

46.- Centers for Disease Control and Prevention (CDC). Update to CDC's sexually transmitted diseases treatment guidelines, 2006: fluoroquinolones no longer recommended for treatment of gonococcal infections. MMWR Morb Mortal Wkly Rep 2007; 56: 332.

47.- Chisholm S A, Mouton J W, Lewis D A, Nichols T, Ison C A, Livermore D M. Cephalosporin MIC creep among gonococci: time for a pharmacodynamic rethink? J Antimicrob Chemother 2010; 65: 2141.

48.- Esposito S, Noviello S, Vanasia A, Venturino P. Ceftriaxone versus other antibiotics for surgical prophylaxis: a meta-analysis. Clin Drug Investig 2004; 24: 29-39.

49.- Woodfield J C, Beshay N, van Rij A M. A meta-analysis of randomized, controlled trials assessing the prophylactic use of ceftriaxone. A study of wound, chest, and urinary infections. World J Surg 2009; 33: 2538-50.

50.- Drusano G L, Ambrose P G, Bhavnani S M, Bertino J S, Nafziger A N, Louie A. Back to the future: using aminoglycosides again and how to 
dose them optimally. Clin Infect Dis 2007; 45: 753-60.

51.- Leibovici L, Vidal L, Paul M. Aminoglycoside drugs in clinical practice: an evidence-based approach. J Antimicrob Chemother 2009; 63: 246-51.

52.- Silva F, Cifuentes M, Pinto M E. Resultados de la vigilancia de susceptibilidad antimicrobiana en Chile: Consolidando una red. Rev Chilena Infectol 2011; 28 (1): 19-27.

53.- Vigilancia de Streptococcus pneumoniae. Boletín del Instituto de Salud Pública Volumen 2 Número 5, 2012. Disponible en http://www. ispch.cl/sites/default/files/Neumo\%2015-062012\%20M.pdf

54.- Hamilton-Miller J M T. Antibiotic resistance from two perspectives: man and microbe. Int $\mathrm{J}$ Antimicrob Agents 2004; 23: 209.

55.- Bantar C, Curcio D, Jasovich A, Bagnulo H, Arango A, Bavestrello L, et al. Neumonía aguda adquirida en la comunidad en adultos: Actualización de los lineamientos para el tratamiento antimicrobiano inicial basado en la evidencia local del Grupo de Trabajo de Sudamérica (Consensur II). Rev Chilena Infectol 2010; (Suppl 1) 27: S9-S27.
56.- Charles P G P, Whitby M, Fuller A J, Stirling R, Wright A A, Korman T M, et al. The etiology of community-acquired pneumonia in Australia: Why penicillin plus doxycycline or a macrolide is the most appropriate therapy. Clin Infect Dis 2008; 46: 1513-21.

57.- Weinstein M P. Rationale for revised penicillin susceptibility breakpoints versus Streptococcus pneumoniae: Coping with antimicrobial susceptibility in an era of resistance. Clin Infect Dis 2009; 48: 1596-600.

58.- Solomkin J S, Mazuski J E, Baron E J, Sawyer R G, Nathens A B, DiPiro J T, et al. Guidelines for the selection of anti-infective agents for complicated intra-abdominal infections. Clin Infect Dis 2003, 37: 997-1005.

59.- Chow A W, Evans G A, Nathens A B, Ball C G, Hansen G, Harding G K M, et al. Canadian practice guidelines for surgical intra-abdominal infections. Can J Infect Dis Med Microbiol 2010; 21: 11-37.

60.- Bailey J A, Virgo K S, DiPiro J T, Nathens A B, Sawyer R G, Mazuski J E. Aminoglycosides for intraabdominal infection: equal to the challenge? Surg Infect 2002; 3: 315-35.

61.- Falagas M E, Matthaiou D K, Karveli E A,
Peppas G. Meta-analysis: randomized controlled trials of clindamycin/aminoglycoside vs. $\beta$-lactam monotherapy for the treatment of intra-abdominal infections. Aliment Pharmacol Ther 2007; 25: 537-56.

62.- Wong P F, Gilliam A D, Kumar S, Shenfine J, O’Dair G N, Leaper D J. Antibiotic regimens for secondary peritonitis of gastrointestinal origin in adults. Cochrane Database Syst Rev 2005; (2): CD004539. DOI:10.1002/14651858.CD004539. pub2.

63.- Deresinski S. In the literature. Treatment of infections due to methicillin susceptible Staphylococcus aureus (MSSA): cephalosporins versus semisynthetic penicillins. Clin Infect Dis 2012; 55: iii-iv.

64.- Leman P, Mukherjee D. Flucloxacillin alone or combined with benzylpenicillin to treat lower limb cellulitis: a randomized controlled trial. Emerg Med J 2005; 22: 342-6.

65.- Talbot T R. Surgical site infections and antimicrobial prophylaxis. Mandell, Douglas and Bennett's Principles and Practice of Infectious Diseases. Mandell GL, Bennett JE, Dolin R, eds.7th ed. Philadelphia: Churchill Livingstone Elsevier; 2010; p 3891-904. 